\title{
Sudden cardiac death after robbery: Homicide or natural death?
}

\author{
Giampiero Bottari $^{\mathrm{a}}$, Silvia Trotta ${ }^{\mathrm{a}, *}$, Andrea Marzullo ${ }^{\mathrm{b}}$, Giovanni Meliota ${ }^{\mathrm{c}}$, \\ Marco Matteo Ciccone ${ }^{\mathrm{d}}$, Biagio Solarino ${ }^{\mathrm{a}}$ \\ ${ }^{a}$ Institute of Legal Medicine, Department of Interdisciplinary Medicine (DIM), University of Bari, Piazza G. Cesare 11, 70124, Bari, Italy \\ ${ }^{\mathrm{b}}$ Pathology Unit, Department of Emergency and Transplantation (DETO), University of Bari, Piazza G. Cesare 11, 70124, Bari, Italy \\ ${ }^{\mathrm{c}}$ Department of Pediatric Cardiology, Giovanni XXIII Pediatric Hospital, Via G. Amendola 207, 70126, Bari, Italy \\ d Section of Cardiovascular Diseases, Department of Emergency and Organ Transplantation (DETO) University of Bari, Piazza G. Cesare 11, 70124, Bari, Italy
}

\section{A R T I C L E I N F O}

\section{Keywords:}

Tako-tsubo

Cardiomyopathy

Robbery

Autopsy

Homicide

\begin{abstract}
A B S T R A C T
Tako-Tsubo is one of a number of rare acquired cardiomyopathies that are characterized by left ventricular dyskinesia and symptomatology typical of acute myocardial infarction (AMI). The most important feature is that the clinical features are triggered by a severe physical or emotional stress. The authors describe the story of a woman, who was brutally assaulted by two men during a house robbery and died from sudden heart failure 8 hours later, after being taken to hospital. External examination revealed no macroscopic alteration of the inner organs, whereas microscopy showed contraction bands with myocardial necrosis, subendocardial and interstitial neutrophil infiltration and fibrosis. These findings were consistent with death due to stress cardiomyopathy even in the absence of previous heart disease. The robbers were convicted of homicide and sentenced to eighteen years in prison.
\end{abstract}

\section{Introduction}

Establishing cause and manner of death is never an easy task for the forensic pathologist, who needs to consider circumstantial data shared with police investigators as well as autopsy and histopathological findings. The complex analysis of scientific evidence should allow the Court to reach the right verdict.

In the following report, a sudden cardiac death after a robbery with traumatic injuries inflicted on a 75-year-old woman is presented. A few hours after the assault the victim showed acute coronary disease syndrome before dying of heart failure. The forensic autopsy findings were consistent with a TakoTsubo-like stress cardiomyopathy. The name refers to the morphological features of the left ventricle due to apical dyskinesia which looks like the Japanese takotsubo, a trap for catching octopuses. TakoTsubo is a form of cardiomyopathy mimicking an acute coronary syndrome with peculiar clinical rather than electrocardiographical features. The clinical features resemble those of a heart attack and the predominant symptom is chest pain, followed by dyspnea and syncope, with concomitant increase of cardiac enzymes, such as creatinine kinase and troponine $\mathrm{T}$, and absence of evident coronary heart disease. This syndrome is usually more prevalent in the female gender and in postmenopausal women, but its cause is unknown. The pathogenesis represents a massive release of catecholamines triggered by a strong physical or emotional stress.

The Court established that death was due to the robbery and the offenders were convicted of homicide.

\section{Case report}

\subsection{History}

A 75-year-old woman, owner of a Jewelry store, was the victim of a robbery as she returned home. Two men tried to force her to open a safe and after her refusal they kicked and punched her face, also administering electric shocks to the entire body with an animal defender.

After the assault, the woman was taken to the Emergency Department (ED) with cranio-facial injuries and electric marks; at this point she started complaining of precordial pain. Her clinical history was negative for previous diseases, and a cardiac examination performed a few months earlier revealed no cardiac alterations.

Health personnel carried out blood tests (Table 1) and an ECG (Fig. 1) that revealed significantly increased levels of Troponin and a

\footnotetext{
* Corresponding author. Department of Interdisciplinary Medicine (DIM), Institute of Legal Medicine, Policlinico di Bari Hospital, University of Bari, Piazza G. Cesare 11, 70124, Bari, Italy.

E-mail address: silvia.trotta89@gmail.com (S. Trotta).
} 
Table 1

Blood tests.

\begin{tabular}{llll}
\hline & ED detection & $\begin{array}{l}\text { CICU detection (after } 3 \\
\text { hours) }\end{array}$ & Normal values \\
\hline Troponin & 2904 & 5627 & $<19 \mathrm{ng} / \mathrm{L}$ \\
CPK & 411 & - & $38-234 \mathrm{IU} / \mathrm{L}$ \\
Mioglobina & 450 & - & $<46 \mu \mathrm{g} / \mathrm{L}$ \\
Hb & 11.6 & 11.3 & $11.4-15.1 \mathrm{~g} / \mathrm{dL}$ \\
RBC & 4.78 & 4.70 & $3.75-5.07 \times 10^{6} /$ \\
& & & $\mu \mathrm{L}$ \\
WBC & 12.12 & 13.32 & $4.0-10.33 \times 10^{3} /$ \\
& & & $\mu \mathrm{L}$ \\
\hline
\end{tabular}

depression of the ST segment.

Therefore, she was admitted to the Cardiac Intensive Care Unit (CICU) with a diagnosis of NSTEMI and administration of antiplatelet and B-blocking drugs. Blood tests were repeated (Table 1). Despite medical treatment, she developed an acute pulmonary edema with pink foam leaking from the mouth and presented episodes of severe bradycardia and electromechanical dissociation despite the administration of inotropic drugs. The patient had a cardio-circulatory arrest and after numerous resuscitation attempts, died approximately 8 hours after the aggression.

\subsection{Autopsy}

On external examination, the face showed numerous traumatic injuries which bore testimony to the violence of the acts, and to the effects of the electrocution (Fig. 2). Many bruises were observed on the frontal and temporal regions with the involvement of the eyelids and nose. Moreover, numerous little abrasions of the epidermidis were noted. Similarly, the lower cervical region, on left side, presented suffused bruises also detectable on the dorsal side of the right hand.

The autopsy revealed tracheal edema and laryngeal hemorrhagic infiltration, multiple rib fractures without macroscopic alteration of the inner organs. The heart, which weighed $390 \mathrm{~g}$, had mild left ventricular dilation with free and septal ventricular wall about $1 \mathrm{~cm}$ thick and flaccid in consistence (Fig. 3a,b,c). A prolapse of the floor of foramen ovalis in the right atrium was observed. The mitral annulus was partially calcific with a sclerotic anterior leaflet. The right coronary artery had a few non-occlusive atherosclerotic plaques. Non-occlusive atherosclerotic plaques were also present in anterior interventricular and circumflex coronary arteries (Fig. 4).

Microscopically myocardial interstitial edema and diffuse fragmentation of myocardial cells with a mild and focal polymorphonuclear leukocyte infiltrate was observed (Fig. 5a). In the wall of the left ventricle, areas of wavy fibers (Fig. 5b) and contraction band necrosis (Fig. 5c) were also found together with mild interstitial and subendocardial fibrosis. Coronary arteries showed circumferential noncomplicated fibro-atheromatous plaques with an intact muscular layer (Fig. 6). The findings were consistent with catecholamine cardiomyopathy, ${ }^{1,2}$ which was ruled as the cause of death. Both autopsy and histopathological findings were far more specific than clinical presentation and ECG pattern observed at the ED.

\section{Discussion}

Is there a relationship between emotional states and heart function? The medical literature is filled with reports of stress-induced cardiac disorders, including sudden cardiac death (SCD). There are many medical denominations, "human stress cardiomyopathy syndrome", "broken heart syndrome", "left ventricular apical ballooning syndrome", "ampulla cardiomyopathy", "apical ballooning syndrome", and "Takotsubo syndrome" (TTS) to cite a few.

Clinically, it is defined as a cardiomyopathy, but it could be considered a syndrome because of common phenotypes caused by multiple pathophysiological pathways and the evidence of disfunction of coronary microcirculation, in the absence of a primitive myocardial pathology. It is prevalent in women, and above all in postmenopausal women. TTS shares more similarities with heart failure, than coronary syndrome, because the major acute complication is pulmonary edema,

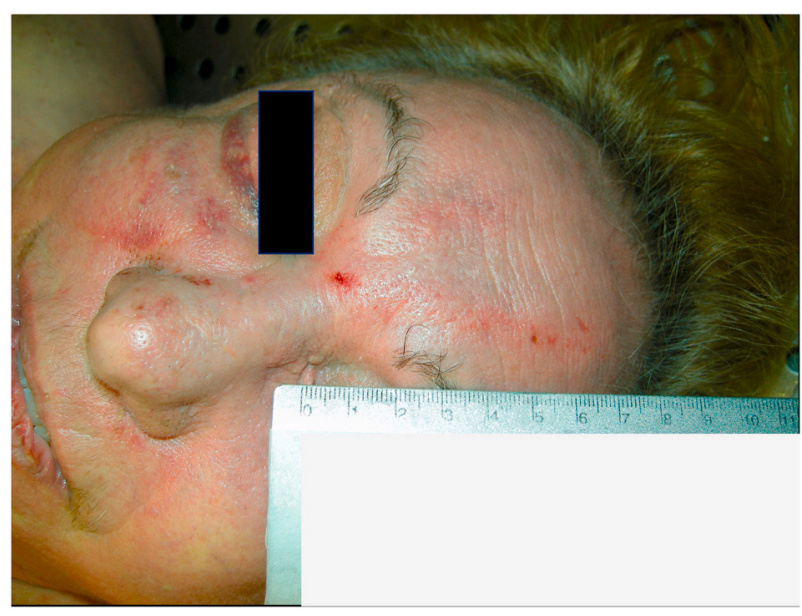

Fig. 2. Victim's face showing traumatic injuries.

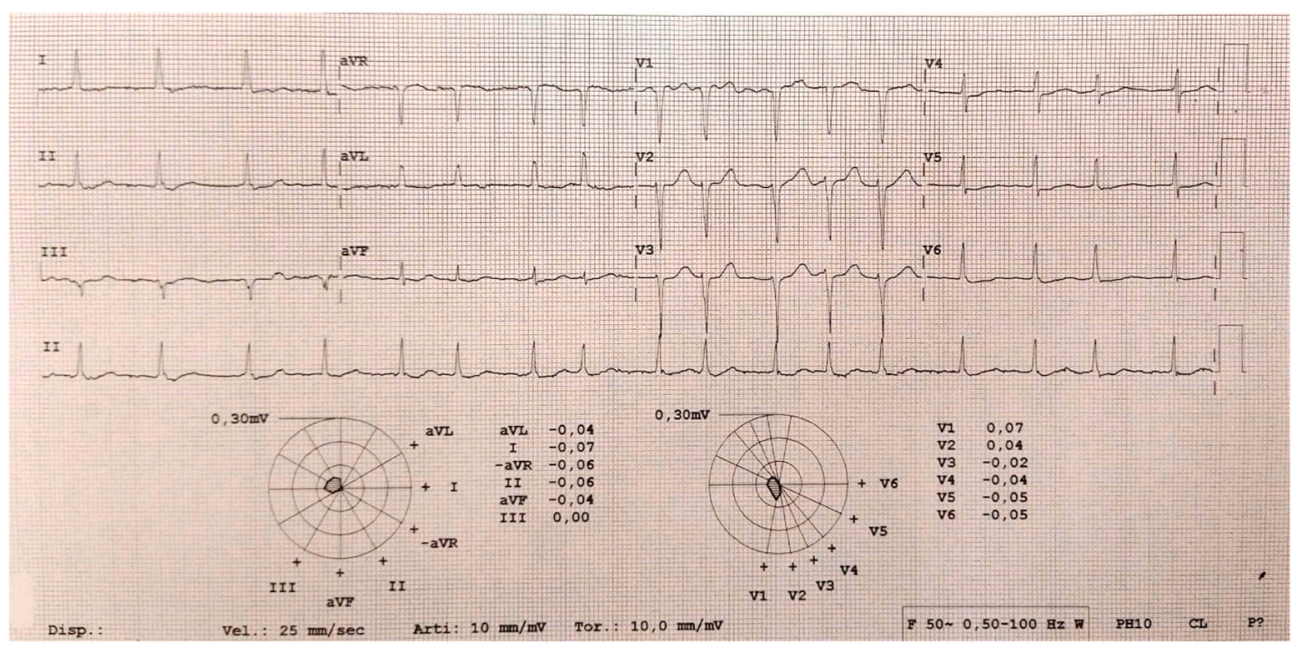

Fig. 1. ECG pattern taken at the ED, showing diffuse ST depression and atrial fibrillation. 
(a)

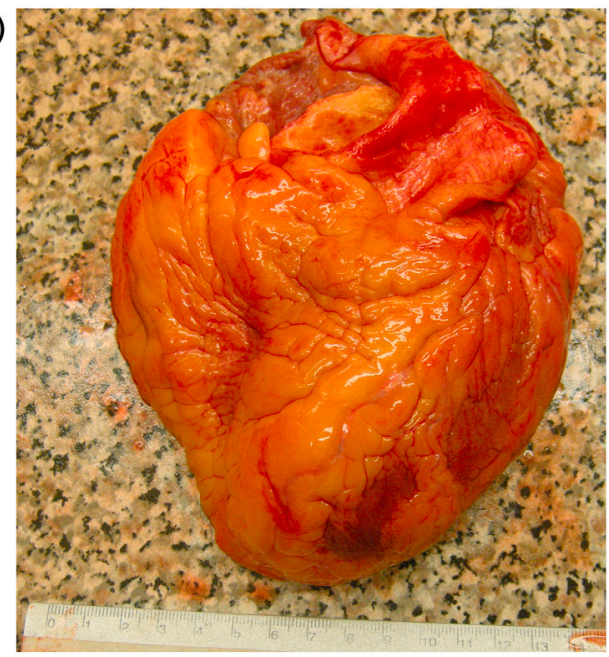

(b)

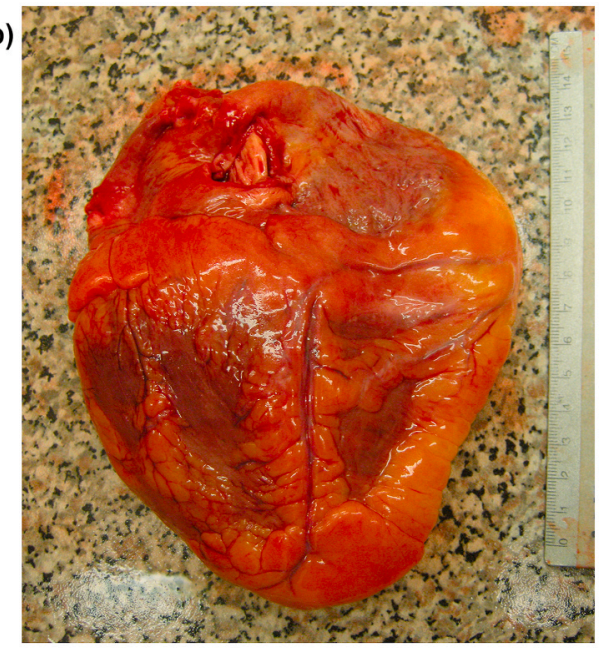

(c)

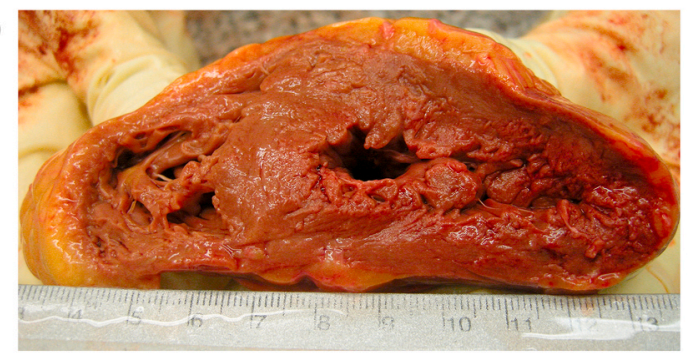

Fig. 3. Macroscopic aspect of the heart: a) anterior wall, b) posterior wall, c) cross section showing mild left ventricular dilation and flaccid in consistence.

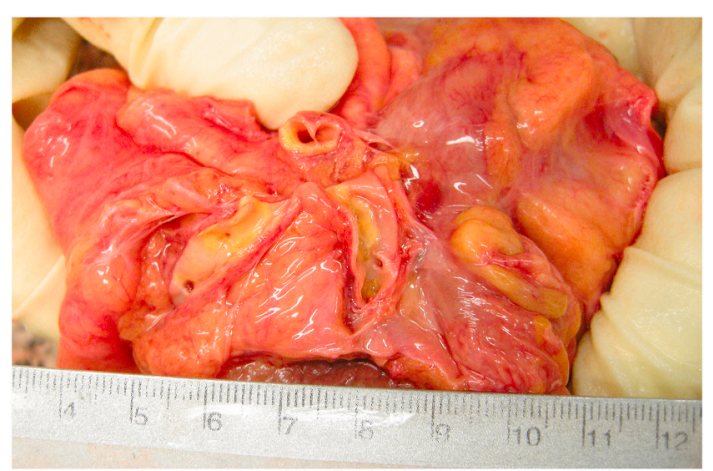

Fig. 4. Left coronary artery showing non-occlusive atherosclerotic plaques.

in the presence of myocardial stunning. The new clinical criteria for diagnosis are edited by HFA-ESC, reviewing the Mayo Clinic criteria. ${ }^{3}$ These include transient anomalies of myocardial kinesis of the left or right ventricle, prior to a stressful event (physical or emotive); usually the dyskinesia extends over the myocardial distribution of a single epicardial vessel, resulting in a circumferential disfunction. Left ventriculography reveals apical and mild ventricular akinesis, apical dilatation with normal contractility of the base of the heart. ${ }^{3-6}$ New and reversible ECG findings are present during the acute phase of pathology (3 months); they are represented by T-wave inversion which usually resolves after approximately 3-4 months, and Q-wave formation, with ST segment changes. Laboratory investigation generally shows elevation of BNP or NT-proBNP, and moderate increase of cardiac enzymes (Troponin). A complete recovery from heart failure after the critical phase is usually observed. ${ }^{3}$

The most important characteristic of TTS is the absence of significant atherosclerotic coronaropathy, inclusive of the rupture of atherosclerotic plaque, thrombosis, coronary dissection, or other cardiomyopathy which interfere with myocardial function (hypertrophic cardiomyopathy, viral myocarditis). The complications include heart failure, cardiogenic shock, ventricular arrhythmia, cardiac tamponade, wall rupture. Sometimes left ventricular outflow tract obstruction (LVOTO) occurs, and it is related to mitral valve failure. The therapy is essentially based on B-Blocker drugs. $^{7}$

TTS could be associated with an emotive trigger, caused by a dramatic event such as the death of a spouse, or fright subsequent to a robbery. Normal daily life events could represent a possible risk for generating an emotive trigger, such as a squabble or disappointment generating ire, and they could occur above all in patients with predisposing clinical conditions, including mood disorders, thyroid disorders, pulmonary disorders. ${ }^{3}$ Maldonado et al. reported a TTS triggered by a maniac episode in patient with bipolar I disorder and history of depression. ${ }^{8}$

Sometimes clinical features are triggered by pain associated with different illnesses, such as pneumothorax, cholecystitis, pancreatitis, bone fractures, or pathologies that trigger a high catecholamine stress or iatrogenic stress due to surgery. In this regard, pheochromocytoma could represent a possible cause of TTS, but myocarditis or hypertrophic cardiomyopathy must be excluded for diagnosis. ${ }^{3}$

The pathogenesis of TTS is not perfectly known, but there is scientific evidence to suggest a possible linkage between the sympathetic nervous system and pathogenesis of several cardiovascular conditions., 90

Certain pathological conditions can interfere with the normal brain heart regulatory mechanism and result in impaired cardiovascular function. ${ }^{11}$

The stress-related cardiomyopathies appear similar as they seemingly occur during times of enhanced sympathetic tone and may be precipitated partly or entirely by excessive endogenous or exogenous catecholamine stimulation of the myocardium. Stress cardiomyopathy can occur after acute mental or physical stress, sub-arachnoid hemorrhage, ischemic stroke, major head trauma, acute medical illness, during pheochromocytoma crisis, and as a result of exogenous catecholamine 
(a)

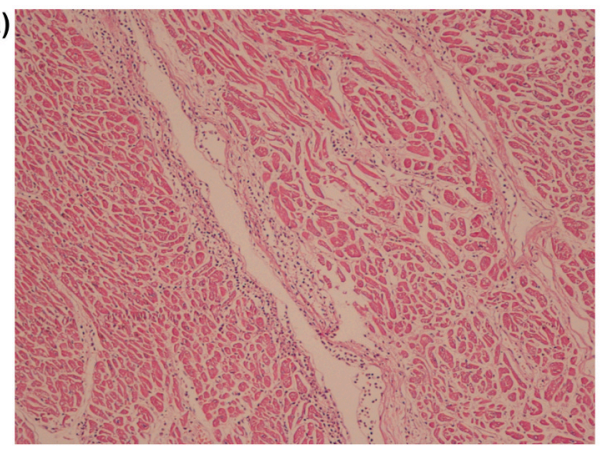

(b)

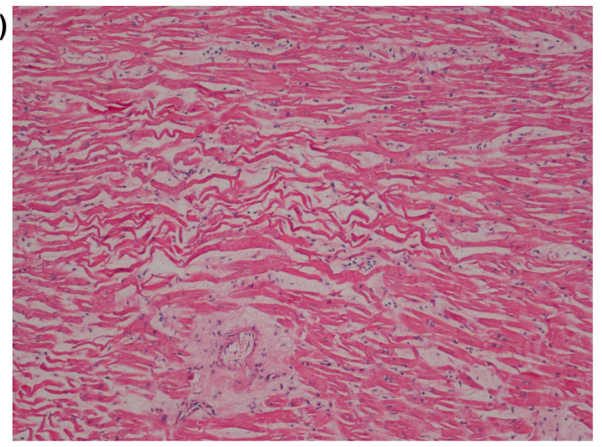

(c)

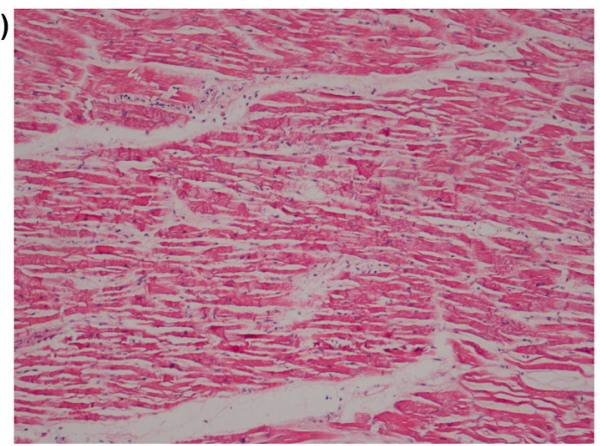

Fig. 5. Histopathology of the left myocardium: a) mild and focal polymorphonuclear leukocyte infiltrate (HE, $40 \times$ ), b) wavy fibers (HE, $100 \times$ ), c) contraction band necrosis $(\mathrm{HE}, 100 \times$ ).

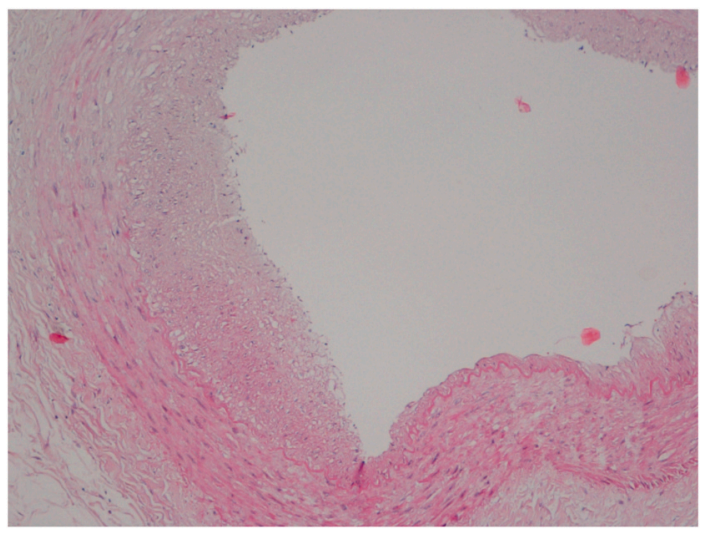

Fig. 6. Left coronary artery showing circumferential non-complicated fibroatheromatous plaques with an intact muscular layer $(\mathrm{HE}, 100 \times)$.

administration. ${ }^{11}$

The histological findings of contraction band necrosis in stress cardiomyopathy suggests a possible common catecholamine - mediated mechanism, but it is unclear whether myocardial adrenergic hyperstimulation is the only pathophysiological mechanism responsible for this syndrome. ${ }^{11}$

There are many clinical similarities between the Neurogenic stress cardiomyopathy (NSC) and TakoTsubo cardiomyopathy (TC). Both conditions appear to be catecholamine mediated. They occur more often in women, present with transient left ventricle wall motion abnormalities in the absence of obstructive coronary artery disease, show ischemic appearing ECG abnormalities, and manifest mild elevations in cardiac biomarkers of myonecrosis. A difference could be observed in low manifestation of isolated apical and mid ventricular wall motion abnormalities in NSC, in patients with TC. Kinetic evidence includes akinesia or dyskinesia of apical and mid-ventricular segments provoking the peculiar apical ballooning visible at the echocardiogram. Thus, many authors associate the myocardial disorder with a toxic effect exerted by high levels of catecholamines which determine the typical fibrosis of the apex of the heart according to the major distribution of $\mathrm{B}$ receptors on this cardiac segment. ${ }^{12}$

The forensic interest is aroused by Sudden cardiac death that could occur subsequent to a criminal act, involving physical and verbal aggression.

The medico-legal aim is to establish a possible correlation between the offender's criminal action and the fatal event. The main aim is to qualify or quantify the extent of emotive reaction in the victim.

The medico-legal evaluation is complicated by any pathologies, above all cardiac or cerebral disorders, with poor prognosis in the mid to long term, and which represent a "pre-existence" status.

These disorders could autonomously cause death, even in the absence of an emotional stress. Thus, the question is the possibility of attributing or denying a causal role to the stressful action that preceded the exitus.

Autopsy findings correlated to TTS are essentially derived from histological examination of myocardial samples. The interstitial infiltrates of mononuclear lymphocytes and macrophages, and contraction bands (CBN) with or without necrosis represent a peculiar histopathological evidence. ${ }^{13,14}$ The inflammatory changes and contraction bands distinguish TTS from coagulation necrosis, as seen in myocardial infarction resulting from coronary artery occlusion. Furthermore, CBN is typical of catecholamine cardiotoxicity, and it is visible within 5-10 minutes of catecholamine infusion. ${ }^{13}$ The histological findings of eosinophilic infiltration, spotty coagulation necrosis, and spotty fibrosis suggest that the pathological changes were subacute and chronic, in fact TCM has been included among cardiomyopathies recently classified which are, by definition, chronic illnesses. ${ }^{15}$

From a forensic standpoint, Davis et al. defined these cases as "homicide by heart attack" and provided such criteria to recognize them establishing a model for determining the cause and manner of death. The criteria include the severity of the criminal act, intending to kill or maim; the victim's understanding of the threat to personal safety; the correlation of the action to a high emotion responses; collapse and death 
occur during the emotional response period (even if the criminal act had already ceased); possible autoptic demonstration of an organic cardiac disease commonly associated with a predisposition to lethal cardiac arrythmia. $^{16}$

In such cases, death may be delayed as a result of medical intervention that would break the chain from organic failure to death. It could be inappropriate to consider homicide when the victim at first recovers to normal vital function, and then deteriorates and dies from heart disease. On the other hand, if the victim was relatively functional prior to the incident and collapse but survived in the intensive care unit for several days prior to death, it would be appropriate to rule the death as homicide, as long as all other criteria are fulfilled. ${ }^{17}$ In this sense, it could be harder to establish the "emotional response period", thus an accurate evaluation of every single case is needed.

Hanzlick et al. proposed including death delayed due to resuscitative efforts. In such cases, more attention is required when evaluating the modifications of the heart found at autopsy. ${ }^{18}$

Furthermore Turner et al. proposed modifying the criteria including contact between the assailant and the victim resulting in injuries, but the injuries must not be life threatening or lethal to conclude that the death was a homicide by heart attack. ${ }^{17}$

The original Davis criteria do not include situations where no physical injury occurs. Leigh Hlavaty et al. recommended including contact that does not result in life-threatening or lethal injuries in the criteria for diagnosing homicide by heart attack. The autopsy analysis revealed that more than $80 \%$ of cases displayed significant changes because of hypertension, causing cardiomegaly and left ventricular hypertrophy. Furthermore, physical contact between the assailant and victims were documented, as evidenced in external injuries and minor internal injuries. ${ }^{19}$

The aim of forensic activity is to determine the "measure" in which a stressful stimulus contributes to cardiovascular disease.

The criminal activity or other act of aggression could precipitate cardiovascular condition to death.

The determination of the Cause and Manner of death represents a complex analysis of autopsy findings systematically coupled with adequate circumstantial data and historical information. ${ }^{20}$

Therefore, sudden cardiovascular death coexisting with a sudden psychologically stressful event is routinely evaluated in clinical medicine and forensic pathology. ${ }^{20}$

The question about the timing of death and the emotional stress related to the criminal act come to the forefront in the Manner of death discussion in this particular case.

Reasonably, the elapsed time from collapse to death allows for the establishment of the probability that stressful events cause the organic failure. The organic deterioration could suggest the continuity from aggression or criminal act to death, even if intensive treatments allow a longer survival time. ${ }^{17}$

Autopsy can demonstrate organic cardiac disease, but it is not sufficient to rule such cases as homicide. In such circumstances, there is no necropsy evidence, microscopic or toxicology results, to recognize the organic defeats and pathologies linked to stressful or violent aggression. For example, a lethal dysrhythmia is a condition with no anatomic correlation, although macroscopic and histopathological myocardial alterations might be related to the genesis of cardiac arrythmias.

A complete autopsy, including histological examination and toxicology, coupled with detailed reconstruction of the events, is essential in order to appropriately certify sudden death.

Takotsubo cardiomyopathy might represent a possible organic pathology which "breaks" or "strengthens" a forensic causality between criminal act and death.

\section{Declaration of competing interest}

The authors declare that they have no conflict of interest.

\section{Acknowledgments}

The authors wish to thank Samantha Austen for her revision and support.

\section{References}

1. Pepe M, Zanna D, Quagliara D, et al. Sudden cardiac death secondary to demonstrated reperfusion ventricular fibrillation in a woman with Takotsubo cardiomyopathy. Cardiovasc Pathol. 2011;20(4):254-257. https://doi.org/10.1016/ j.carpath.2010.06.006.

2. Mitchel A, Marquis F. Can Tako Tsubo cardiomyopathy be diagnosed at autopsy? Report of a presumed case presenting as cardiac rupture. BMC Clin Pathol. 2017;17: 4. https://doi.org/10.1186/s12907-017-0045-0.

3. Lyon AR, Bossone E, Schneider B, et al. Current state of knowledge on takotsubo syndrome: a position statement from the taskforce on takotsubo syndrome of the heart failure association of the European society of cardiology. Eur $J$ Heart Fail. 2016;18(1):8-27. https://doi.org/10.1002/ejhf.424.

4. Bounhoure JP. Takotsubo or stress cardiomyopathy. Cardiovasc Psychiatry Neurol. 2012;2012:637672. https://doi.org/10.1155/2012/637672.

5. Kawai S, Suzuki H, Yamaguchi H, et al. Ampulla cardiomyopathy ('Takotusbo' cardiomyopathy) - reversible left ventricular dysfunction with ST segment elevation. Jpn Circ J. 2000;64(2):156-159. https://doi.org/10.1253/jcj.64.156.

6. Bybee KA, Kara T, Prasad A, et al. Systematic review: transient left ventricular apical ballooning: a syndrome that mimics ST-segment elevation myocardial infarction. Ann Intern Med. 2004:858-865. https://doi.org/10.7326/0003-4819-141-11200412070-00010.

7. Wittstein IS, Thiemann DR, Lima JAC, et al. Neurohumoral features of myocardial stunning due to sudden emotional stress. N Engl J Med. 2005;352(6):539-548. https://doi.org/10.1056/NEJMoa043046.

8. Maldonado JR, Pajouhi P, Witteles R. Broken heart syndrome (Takotsubo Cardiomyopathy) triggered by acute mania: a review and case report. Psychosomatics. 2013;54:74-79. https://doi.org/10.1016/j.psym.2012.03.009.

9. Samuels MA. The brain-heart connection. Circulation. 2007;116:77-84. https://doi. org/10.1161/CIRCULATIONAHA.106.678995.

10. Dijkhuizen LGM, Kubat B, Duijst WLJM. Sudden death during physical restraint by the Dutch police. J Forensic Leg Med. 2020;72:101966. https://doi.org/10.1016/j. jflm.2020.101966.

11. Bybee KA, Prasad A. Stress-related cardiomyopathy syndromes. Circulation. 2008; 118:397-409. https://doi.org/10.1161/CIRCULATIONAHA.106.677625.

12. Wybraniec M, Mizia-Stec K, Krzych $€$. Stress cardiomyopathy: yet another type of neurocardiogenic injury: "Stress cardiomyopathy". Cardiovasc Pathol. 2014;23: 113-120. https://doi.org/10.1016/j.carpath.2013.12.003.

13. Akashi YJ, Goldstein DS, Barbara G, Ueyama T. Takotsubo cardiomyopathy a new form of acute, reversible heart failure. Circulation. 2008;118:2754-2762. https:// doi.org/10.1161/CIRCULATIONAHA.108.767012.

14. Kinbara T, Hayano T, Otani N, Furutani Y, Murakami T, Yano M. An autopsy case of Tako-Tsubo cardiomyopathy presenting ventricular tachycardia after pacemaker implantation. J Cardiol Cases. 2013;8:134-137. https://doi.org/10.1016/j. jccase.2013.06.007.

15. Indorato F, Bartoloni G. Post-mortem Takotsubo cardiomyopathy diagnosis: the challenge is open!. Forensic Sci Med Pathol. 2016;12:227-228. https://doi.org/ 10.1007/s12024-016-9759-z.

16. Davis JH. Can sudden cardiac death be murder? J Forensic Sci. 1978;23(2):384-387. https://doi.org/10.1520/jfs10773j.

17. Turner SA, Barnard JJ, Spotswood SD, Prahlow JA. Homicide by heart attack revisited. J Forensic Sci. 2004;49(3):598-600. https://doi.org/10.1520/jfs2003343.

18. Hanzlick R, Hunsaker JH, Davis GJ. A Guide for Manner of Death Classification. St. Louis, MO: National Association of Medical Examiners; 2001.

19. Hlavaty L, Sung L. Applying the principles of homicide by heart attack. Am J Forensic Med Pathol. 2016;37(2):112-117. https://doi.org/10.1097/ PAF.0000000000000232.

20. Solarino B, Ralston W, Younger K, Hunsaker DM. Sudden natural death in a suicide attempt. Forensic Sci Med Pathol. 2006;2:189-192. https://doi.org/10.1007/s12024006-0008-8. 